\title{
Estimation with unknown inputs and uncertainties for sampled-data systems based on quasi sliding mode
}

DOI:

10.1080/00207179.2020.1750706

\section{Document Version}

Accepted author manuscript

Link to publication record in Manchester Research Explorer

\section{Citation for published version (APA):}

Nguyen, T., Edwards, C., \& Herrmann, G. (2020). Estimation with unknown inputs and uncertainties for sampleddata systems based on quasi sliding mode. International Journal of Control, 0, 1-11.

https://doi.org/10.1080/00207179.2020.1750706

\section{Published in:}

International Journal of Control

\section{Citing this paper}

Please note that where the full-text provided on Manchester Research Explorer is the Author Accepted Manuscript or Proof version this may differ from the final Published version. If citing, it is advised that you check and use the publisher's definitive version.

\section{General rights}

Copyright and moral rights for the publications made accessible in the Research Explorer are retained by the authors and/or other copyright owners and it is a condition of accessing publications that users recognise and abide by the legal requirements associated with these rights.

\section{Takedown policy}

If you believe that this document breaches copyright please refer to the University of Manchester's Takedown Procedures [http://man.ac.uk/04Y6Bo] or contact uml.scholarlycommunications@manchester.ac.uk providing relevant details, so we can investigate your claim.

\section{OPEN ACCESS}




\title{
ARTICLE TEMPLATE
}

\section{Estimation with unknown inputs and uncertainties for sampled-data systems based on quasi sliding mode}

\author{
Thang Nguyen ${ }^{\mathrm{a}}$ and Christopher Edwards ${ }^{\mathrm{b}}$ and Guido Herrmann ${ }^{\mathrm{c}}$ \\ a Modeling Evolutionary Algorithms Simulation and Artificial Intelligence, Faculty of \\ Electrical \& Electronics Engineering, Ton Duc Thang University, Ho Chi Minh City, \\ Vietnam; ${ }^{\mathrm{b}}$ College of Engineering, Mathematics and Physical Sciences, University of Exeter, \\ Exeter, UK, EX4 4QF, UK; ' Department of Electrical and Electronic Engineering, The \\ University of Manchester, Sackville St Building, Manchester, M13 9PL, UK.
}

\author{
ARTICLE HISTORY \\ Compiled February 8, 2021
}

\begin{abstract}
In this work, we consider the problem of simultaneously estimating the system states and unknown inputs in a linear sampled-data system, whose dynamics is influenced by external disturbances and uncertainties. Hardware limitations prevent an estimation scheme for a sampled-data system from achieving finite-time convergence, which is a typical property of existing sliding mode observers for dynamical continuoustime systems, because the sampling period is finite. Due to the sampling process, an approximate implementation of such an observer, designed for a continuous-time system, may not retain the desired performance in the sampled-data context. In this paper, we present an observer which takes advantage of the quasi-sliding motion concept to simultaneously estimate the state variables and the unknown input signals in a sampled-data context. A theoretical study is conducted to formally justify the convergence properties of the observer whilst simulation results are provided to show the efficiency of the proposed scheme.
\end{abstract}

\section{KEYWORDS}

Sliding mode observer; sampled-data systems; unknown inputs; disturbances; uncertainty.

\section{Introduction}

There is significant literature which discusses sliding mode approaches for designing observers to simultaneously estimate system states and unknown inputs for continuoustime systems: for example see (Alwi \& Edwards, 2008; Bejarano \& Fridman, 2010; Chandra, Alwi, \& Edwards, 2017; Edwards, Spurgeon, \& Patton, 2000; Floquet, Edwards, \& Spurgeon, 2007; Gucik-Derigny, Raïssi, \& Zolghadri, 2016; Tan \& Edwards, 2003; Yan \& Edwards, 2007) and the references therein for a class of observers in which the system representing the dynamics between the disturbance inputs and the measurements is relative degree one. A common procedure in designing such observers includes (i) first selecting a sliding surface which depends on the output errors; (ii) the use of a nonlinear switching injection gain to force the output errors to reach the sliding plane in finite time. The unknown input signals are then recovered in a robust 
and reliable manner through the nonlinear injection signal (typically through the use of low-pass filters).

There have been a variety of papers which address the corresponding estimation problem for discrete-time systems with unknown inputs; see for example (Chakrabarty, Ayoub, Żak, \& Sundaram, 2017; Charandabi \& Marquez, 2014; Floquet \& Barbot, 2006; Marro, Bernstein, \& Zattoni, 2010; Marro, Zattoni, \& Bernstein, 2011; Sundaram \& Hadjicostis, 2007). In (Floquet \& Barbot, 2006), a sampling delayed observer is proposed under the assumption that the system does not have invariant zeros and is left-invertible. In this work, the delayed estimator is designed using a change of coordinates which creates a canonical form well-suited for its construction. Some variables that are independent of the unknown inputs are constructed, and then the states and unknown input signals of the linear systems are reliably inferred after a certain (finite) number of sampling delays. In (Sundaram \& Hadjicostis, 2007), a delayed estimator scheme is proposed to simultaneously recover the unknown inputs and unknown state variables of a discrete-time system, where the unknown inputs are separated from the estimation error using a parameterization of the observer tuning parameter. In (Marro et al., 2010, 2011), geometric methods are introduced to recover both the state variables and the unknown inputs for a class of discrete-time linear systems. In these papers, a finite impulse response (FIR) system is employed to process the output measurements. Accordingly, the initial state and the subsequent state values are recovered with a delay which depends on the characteristics of the original system. The unknown input is then subsequently reconstructed using another delay of one step. In (Charandabi \& Marquez, 2014), an $H_{\infty}$ filter method is presented for discrete-time linear systems with unknown inputs without resorting to similarity transformations. A linear filter is constructed based on the original model to extract both the original states and the unknown inputs of the system. The work in (Chakrabarty et al., 2017) used linear matrix inequalities (LMIs) to design a delayed observer whose tuning parameters and peak-gain bounds on a prescribed performance index of the estimator are computed. In this work, the unknown inputs can be recovered to a designated degree of accuracy under the conditions that the system possesses the minimum-phase property and the designated delay is large enough.

The aforementioned discussion demonstrates considerable literature on observers for continuous-time and discrete-time systems, however in comparison, not much research has been published in the sampled-data context for observers which are derived from sliding mode or quasi-sliding mode ideas ${ }^{1}$. This class of systems is important because even if the observer is designed from a model in the continuous time domain, it is invariably implemented digitally. In sampled-data systems, there is no continuity in the output measurement which is taken at sampling instants, and in addition, the system behavior between sampling instants should be considered in the sampled-data context - which differs from discrete-time systems. In (Han, Fridman, \& Spurgeon, 2014), the problem of robust fault recovery for a class of continuous systems is addressed in which the output signals are measured at the sampling instants but the control input is still continuous. It is well understood that while theoretically sliding mode systems require infinite frequency switching, sampled-data systems cannot possess such capabilities due to the sample/hold operation. Hence, no ideal sliding motion exists, and only socalled "quasi sliding modes" can be achieved in which the system state is kept in a boundary layer of the sliding plane (Milosavljevic, 1985).

\footnotetext{
${ }^{1}$ Many of the higher order sliding mode observers/differentiators of Levant and his colleagues do describe performance bounds resulting from the practicalities of discrete implementation (Fridman, Levant, \& Davila, 2007; Levant, 2011; Livne \& Levant, 2014).
} 
In this work, we propose an observer to address the problem of simultaneous system state and unknown input estimation for a sampled-data system with uncertainties. The proposed scheme is developed based on quasi-sliding motion concept under the assumption that the continuous time system is minimum-phase and has relative degree one. Our work and other papers in the literature differ in the fact that we employ a one-step delay for the observer designed for sampled-data systems which consider the sampling time period. The contribution of our work is as follows. A novel sampleddata estimator is constructed to simultaneously recover system state and unknown disturbance signals for a class of sampled-data systems in the presence of uncertainties. A theoretical analysis on convergence and robustness is given to support the proposed method.

Throughout the paper, the symbol $R$ represents the field of real numbers and $\lambda\{A\}$ stands for the spectrum of the matrix $A$. The symbol $I_{m}$ denotes an identity matrix of order $m$. For a vector, $\|$.$\| represents the Euclidean norm, and for matrices it denotes$ the induced spectral norm. As in (Kokotovic, Khalil, \& O'Reilly, 1986), a vector function $f(t, s) \in R^{n}$ is said to be $O(s)$ over an interval $\left[t_{1}, t_{2}\right]$, if positive constants $K$ and $s^{*}$ exist such that $\|f(t, s)\| \leq K s, \quad \forall s \in\left[0, s^{*}\right], \quad \forall t \in\left[t_{1}, t_{2}\right]$. Finally, the notation $f[k]$ denotes $f\left(k T_{s}\right)$, where $k=0,1,2, \ldots$ stands for the index of the discrete-time sequence obtained due to sampling (where $T_{s}$ is the sampling time period).

The paper is structured as follows: Section 2 presents some preliminaries and describes the problem formulation. Section 3 contains the main results and describes the estimator design. A numerical example is used to show the efficiency of the proposed method in Section 4. Finally, Section 5 provides some concluding remarks.

\section{Preliminaries and Problem Formulation}

In this section, we recall the problem of constructing a conventional sliding mode estimator for a continuous time linear system subject to external disturbances (to be reconstructed) and uncertainties in (Tan \& Edwards, 2003).

Consider the following uncertain LTI system given by

$$
\begin{aligned}
\dot{x}(t) & =A x(t)+B u(t)+F f(t)+M \xi(t, y, u) \\
y(t) & =C x(t),
\end{aligned}
$$

where $x(t) \in R^{n}$ denotes the system state vector, $u(t) \in R^{m}$ stands for the control input vector, $y(t) \in R^{p}$ is the system output vector, $f(t) \in R^{q}$ is an unknown bounded external disturbance (to be reconstructed), $\xi(t, y, u) \in R^{\kappa}$ is lumped uncertainty, the dimensions $q \leq p<n$, and the matrices $C$ and $F$ have full rank. The system matrices $A, B, C, F$, and $M$ in (1) are constant and have appropriate dimensions. In this paper, we assume that the system state vector in (1) is unknown and only the information from control input vector $u(t)$ and output vector $y(t)$ are available.

A sliding mode observer for the system (1) (in the context of a fault detection and isolation problem) was reported in (Tan \& Edwards, 2003). For completeness, we reproduce the procedure to design a sliding mode estimator (Tan \& Edwards, 2003).

Assumption 1. System (1) possesses the following properties:

- $\operatorname{rank}(C F)=q$

- The invariant zeros (if any) of $(A, F, C)$ have negative real parts. 
Assumption 2. The disturbance $f(t)$ and its first and second time derivatives are bounded.

Assumption 3. The function $\xi(t, u, y)$ is an unknown but bounded uncertainty which satisfies

$$
\|\xi(t, y, u)\|<\bar{\xi}
$$

where $\bar{\xi}$ is a known positive scalar.

Remark 1. Assumption 1 represents necessary and sufficient conditions for the design and analysis in (Tan \& Edwards, 2003). The assumptions on the derivatives of $f(t)$ (Assumption 2) are not required in (Tan \& Edwards, 2003) but are required for the developments which follow. The class of functions $f(t)$ can be extended to the case where only the first derivative of $f(t)$ exists and is bounded. The restrictions on the uncertainty $f(t)$ in the sampled-data model follow those in (Nguyen, Edwards, Azimi, $\& \mathrm{Su}, 2019)$.

Remark 2. Assumption 1 represents relative degree one minimum phase conditions for the triple $(A, F, C)$. These are necessary and sufficient conditions for the existence of linear unknown input observers for the continuous-time system $(A, F, C)$ (Darouach, Zasadzinski, \& Xu, 1994). They are also the basis for the work in (Tan \& Edwards, 2003). The rank condition was later obviated using a cascade of sliding mode observers (Tan \& Edwards, 2010).

If the rank condition in Assumption 1 holds and $p>q$, then, without of loss of generality, the triple $(A, F, C)$ can be converted into the following form through state transformation (Tan \& Edwards, 2003):

$$
A=\left[\begin{array}{ll}
A_{11} & A_{12} \\
A_{21} & A_{22}
\end{array}\right], \quad F=\left[\begin{array}{c}
0 \\
F_{0}
\end{array}\right], \quad C=\left[\begin{array}{ll}
0 & T_{0}
\end{array}\right]
$$

where $A_{11} \in R^{(n-p) \times(n-p)}, F_{0} \in R^{p \times q}$ is nonsingular, $T_{0} \in R^{p \times p}$ is orthogonal, and

$$
F_{0}=\left[\begin{array}{c}
0 \\
F_{2}
\end{array}\right]
$$

where $F_{2} \in R^{q \times q}$ and $\operatorname{det} F_{2} \neq 0$. When $p=q, F_{0}=F_{2}$. In (Tan \& Edwards, 2003), a state observer was proposed in the form

$$
\begin{aligned}
\dot{z}(t) & =A z(t)+B u(t)-G_{l} e_{y}(t)+G_{n} v_{n} \\
y_{0}(t) & =C z(t)
\end{aligned}
$$

where

$$
v_{n}= \begin{cases}-\rho(t, y, u) \frac{P_{0} e_{y}}{\left\|P_{0} e_{y}\right\|} & \text { if } e_{y} \neq 0 \\ 0 & \text { otherwise }\end{cases}
$$

is a discontinuous output error injection vector. Here $e_{y}:=y_{0}-y$ represents the output estimation error and the function $\rho: R_{+} \times R^{p} \times R^{m} \rightarrow R$ is a scalar design parameter. 
In (Tan \& Edwards, 2003), the matrices $P_{0}, G_{n}$, and $G_{l}$ are obtained from the solution of an $\mathcal{L}_{2}$ gain optimization problem (Tan \& Edwards, 2003).

In the sampled-data context, the control input signal $u(t)$ is held constant between consecutive sampling instants and the output is measured at sampling times. Thus, the direct implementation of the observer in (5) as it stands may not be suitable (see the example in Section 4 later in the paper). In addition, the system hardware limitations, or more general system wide constraints, may prevent the sample time period $T_{s}$ from being as small as the designer would like, and the infinite switching mechanism inherent in (6) to recover the unknown disturbance signal $f(t)$ cannot be realised. This motivates the development of an estimator for a sampled-data version of system (1) to estimate the state variables and the unknown input signal $f(t)$ simultaneously despite the presence of the uncertainty in $\xi(t)$.

The minimum phase condition on $(A, F, C)$ in Assumption 1 ensures the pair $\left(A_{11}, A_{211}\right)$ is detectable where $A_{211}$ represents the top $p-q$ rows of $A_{21}$ (Tan \& Edwards, 2003). Consequently, there exists an $L=\left[\begin{array}{ll}L_{0} & 0\end{array}\right]$ where $L_{0} \in \mathrm{R}^{n-p \times p-q}$ such that $\mathcal{A}_{11}=A_{11}+L A_{21}$ is stable. The following transformation $x \rightarrow T_{L} x$ (Tan \& Edwards, 2003) based on $L$ helps facilitate the design and analysis procedure

$$
T_{L}=\left[\begin{array}{cc}
I_{n-p} & L \\
0 & T_{0}
\end{array}\right]
$$

The system matrices in the new coordinates take the following structure

$$
\begin{aligned}
\mathcal{A} & =T_{L} A T_{L}^{-1}=\left[\begin{array}{ll}
\mathcal{A}_{11} & \mathcal{A}_{12} \\
\mathcal{A}_{21} & \mathcal{A}_{22}
\end{array}\right], \mathcal{F}=T_{L} F=\left[\begin{array}{c}
0 \\
\mathcal{F}_{2}
\end{array}\right], \mathcal{C}=C T_{L}^{-1}\left[\begin{array}{ll}
0 & I_{p}
\end{array}\right], \\
\mathcal{M} & =T_{L} M=\left[\begin{array}{l}
\mathcal{M}_{1} \\
\mathcal{M}_{2}
\end{array}\right], \mathcal{B}=T_{L} B,
\end{aligned}
$$

where $\mathcal{A}_{11}=A_{11}+L A_{21}$ and $\mathcal{F}_{2}=T_{0} F_{2}$. In the new coordinates, the system in (1) becomes

$$
\begin{aligned}
\dot{\bar{x}}(t) & =\mathcal{A} \bar{x}(t)+\mathcal{B} u(t)+\mathcal{F} f(t)+\mathcal{M} \xi(t, y, u) \\
y(t) & =\mathcal{C} \bar{x}(t),
\end{aligned}
$$

where $\bar{x}=T_{L} x$ is the new state vector. The sampled-data representation of (9) is

$$
\begin{aligned}
\bar{x}[k+1] & =\Phi \bar{x}[k]+\Gamma u[k]+d[k]+\beta[k] \\
y[k] & =C \bar{x}[k]
\end{aligned}
$$

where the constant matrices $\Phi=e^{\mathcal{A} T_{s}}$ and $\Gamma=\int_{0}^{T_{s}} e^{\mathcal{A} \tau} d \tau \mathcal{B}$. In (10), the external disturbance is

$$
d[k]=\int_{0}^{T_{s}} e^{\mathcal{A} \tau} \mathcal{F} f\left((k+1) T_{s}-\tau\right) d \tau,
$$

and the modelling uncertainty is

$$
\beta[k]=\int_{0}^{T_{s}} e^{\mathcal{A} \tau} \mathcal{M} \xi(\bar{\tau}, y(\bar{\tau}), u(\bar{\tau})) d \tau,
$$


where $\bar{\tau}=(k+1) T_{s}-\tau$ and $T_{s}$ is the sampling period. Using the same approach as in (Nguyen, Su, \& Gajic, 2010; Nguyen, Su, Gajic, \& Edwards, 2016), we define $\overline{\mathcal{A}}$ and $\overline{\overline{\mathcal{A}}}$ as

$$
\begin{aligned}
\overline{\mathcal{A}} & =\frac{1}{T_{s}}\left(\Phi-I_{n}\right) \\
\overline{\overline{\mathcal{A}}} & =\frac{1}{T_{s}^{2}}\left(\Phi-I_{n}-T_{s} \mathcal{A}\right) .
\end{aligned}
$$

As a result,

$$
\overline{\mathcal{A}}=\mathcal{A}+T_{s} \overline{\overline{\mathcal{A}}}
$$

and because

$$
\Phi=\sum_{k=0}^{\infty} T_{s}^{k} \frac{\mathcal{A}^{k}}{k !},
$$

and

$$
\overline{\overline{\mathcal{A}}}=\sum_{k=2}^{\infty} T_{s}^{k-2} \frac{\mathcal{A}^{k}}{k !}=O(1)
$$

it follows that

$$
\Phi=I_{n}+T_{s}\left(\mathcal{A}+T_{s} \overline{\overline{\mathcal{A}}}\right) .
$$

Define

$$
\Pi=\int_{0}^{T_{s}} e^{\mathcal{A} \tau} d \tau \mathcal{F}
$$

and

$$
\overline{\mathcal{F}}=\frac{\Pi}{T_{s}},
$$

and

$$
\overline{\overline{\mathcal{F}}}=\frac{1}{T_{s}^{2}}\left(\Pi-T_{s} \mathcal{F}\right)
$$

Thus, using the same argument as in (Nguyen et al., 2016), we have

$$
\Pi=T_{s} \overline{\mathcal{F}}=T_{s}\left(\mathcal{F}+T_{s} \overline{\overline{\mathcal{F}}}\right)=T_{s}\left(\mathcal{F}+T_{s} \overline{\overline{\mathcal{F}}}\right)=O\left(T_{s}\right) .
$$

Decompose $\overline{\mathcal{F}}$ and $\overline{\mathcal{F}}$ into $\overline{\mathcal{F}}_{1}, \overline{\mathcal{F}}_{2}, \overline{\mathcal{F}}_{1}, \overline{\mathcal{F}}_{2}$, which are conformable to $\mathcal{F}$ in $(8)$. The forms of $\mathcal{F}$ and $\Pi$ in (8) and (22) imply that

$$
\Pi=\left[\begin{array}{c}
T_{s}^{2} \overline{\overline{\mathcal{F}}}_{1} \\
T_{s} \overline{\mathcal{F}}_{2}
\end{array}\right] .
$$


With a small abuse of notation, let

$$
f[k+0.5]=\frac{f[k]+f[k+1]}{2} .
$$

The following lemma describes some characteristics of the unknown signal $d[k]$ : for details see (Nguyen et al., 2016).

Lemma 2.1. If Assumption 2 is satisfied, then

$$
\begin{aligned}
d[k] & =\Pi f[k]+\frac{T_{s}}{2} \Pi v[k]+T_{s}^{3} \Delta d_{0}[k] \\
& =\Pi \frac{(f[k]+f[k+1])}{2}+T_{s}^{3} \Delta d[k] \\
& =\Pi f[k+0.5]+T_{s}^{3} \Delta d[k], \\
d[k] & -d[k-1]=O\left(T_{s}^{2}\right), \\
d[k] & -2 d[k-1]+d[k-2]=O\left(T_{s}^{3}\right),
\end{aligned}
$$

where $v(t)=\dot{f}(t)$, and

$$
\Delta d[k]=\Delta d_{0}[k]-\frac{\Pi}{2} \int_{k T_{s}}^{(k+1) T_{s}} \int_{k T_{s}}^{\sigma} \dot{v}(\tau) d \sigma d \tau,
$$

where

$$
\Delta d_{0}[k]=G v[k]+\frac{1}{T_{s}^{3}} \int_{0}^{T_{s}} e^{\mathcal{A} \tau} \mathcal{F} \int_{k T_{s}}^{(k+1) T_{s}-\tau} \int_{k T_{s}}^{\beta} \dot{v}(\sigma) d \sigma d \beta d \tau,
$$

and

$$
G=\left(-\frac{1}{12} \mathcal{A}-\frac{T_{s}}{12} \overline{\overline{\mathcal{A}}}\right) \mathcal{F}=O(1)
$$

Proof: See the Appendix.

The main results will be presented in the next section in which the purpose is to design a sliding mode estimator for the sampled-data system (10) that produces the estimates of $x[k]$, and recovers the unknown input signal $f[k]$.

\section{Main results}

In this section, the details of the estimator for the sampled-data system in (10) will be presented. The convergence properties of the proposed observer will be studied initially in the absence of the disturbance and uncertainty, then separately, the analysis of the disturbance estimate and state estimates will be conducted. 


\subsection{Observer design}

From Section 2, we can write

$$
\Phi=\left[\begin{array}{ll}
\Phi_{11} & \Phi_{12} \\
\Phi_{21} & \Phi_{22}
\end{array}\right], \quad \Pi=\left[\begin{array}{c}
0 \\
\Pi_{2}
\end{array}\right], \quad C=\left[\begin{array}{ll}
0 & C_{2}
\end{array}\right],
$$

where $\Phi_{11} \in R^{(n-p) \times(n-p)}, \Pi_{2} \in R^{q \times q}$, and $C_{2} \in R^{p \times p}$. Based on these partitions, the observer state $z[k]$ in a partitioned form is

$$
z[k]=\left[\begin{array}{l}
z_{1}[k] \\
z_{2}[k]
\end{array}\right]
$$

where $z_{1}[k] \in R^{n-p}$ and $z_{2}[k] \in R^{p}$. Then it follows that

$$
\begin{aligned}
& z_{1}[k+1]=\Phi_{11} z_{1}[k]+\Phi_{12} y[k]+\Gamma_{1} u[k] \\
& z_{2}[k+1]=\Phi_{21} z_{1}[k]+\Phi_{22} y[k]+\Gamma_{2} u[k]+w[k]
\end{aligned}
$$

where the specifics of the injection signal $w[k]$ will be determined in the sequel.

Let the sampled state estimation error be $e[k]=z[k]-\bar{x}[k]$ and decompose it as

$$
e[k]=\left[\begin{array}{l}
e_{1}[k] \\
e_{2}[k]
\end{array}\right] \text {. }
$$

The output estimation error at the sample times is given by $e_{y}[k]=e_{2}[k]=z_{2}[k]-y[k]$. From Lemma 2.1, and equations (10) and (23),

$$
\begin{aligned}
{\left[\begin{array}{l}
e_{1}[k+1] \\
e_{2}[k+1]
\end{array}\right]=} & {\left[\begin{array}{ll}
\Phi_{11} & 0 \\
\Phi_{21} & 0
\end{array}\right]\left[\begin{array}{l}
e_{1}[k] \\
e_{2}[k]
\end{array}\right]-\left[\begin{array}{c}
T_{s}^{2} \overline{\mathcal{F}}_{2} \\
T_{s} \overline{\mathcal{F}}_{2}
\end{array}\right] f[k+0.5] } \\
& -T_{s}^{3} \Delta d[k]-\beta[k]+\left[\begin{array}{c}
0 \\
w[k]
\end{array}\right]
\end{aligned}
$$

where $\Delta d[k]$ and $\beta[k]$ are conformably partitioned as

$$
\begin{aligned}
\Delta d[k] & =\left[\begin{array}{l}
\delta d_{1}[k] \\
\delta d_{2}[k]
\end{array}\right] \\
\beta[k] & =\left[\begin{array}{l}
\beta_{1}[k] \\
\beta_{2}[k]
\end{array}\right] .
\end{aligned}
$$

The expression in (18) contains the system matrix component

$$
\Phi_{11}=I_{n-p}+T_{s}\left(\mathcal{A}_{11}+T_{s} \overline{\overline{\mathcal{A}}}_{11}\right) .
$$

Because by construction the real parts of the eigenvalues of $\mathcal{A}_{11}=A_{11}+L A_{21}$ are negative (Tan \& Edwards, 2003), there exists a $T_{s}$ such that the spectrum of $\Phi_{11}$ is in the unit circle, i.e. $0<\lambda\left(\Phi_{11}\right)<1$ (see the arguments in (Nguyen et al., 2016) for more details). Denote

$$
\zeta[k]=-T_{s}^{2} \overline{\mathcal{F}}_{2} f[k+0.5]-T_{s}^{3} \delta d_{1}[k]-\beta_{1}[k],
$$


then from (33),

$$
e_{1}[k+1]=\Phi_{11}^{k+1} e_{1}[0]+\sum_{i=0}^{i=k} \Phi_{11}^{i} \zeta[k-i] .
$$

Let $\alpha=\left\|\Phi_{11}\right\|=\max \left(\lambda\left(\Phi_{11}\right)\right)<1$, then we have

$$
\begin{aligned}
\lim _{k \rightarrow \infty}\left\|e_{1}[k+1]\right\| & \leq \lim _{k \rightarrow \infty}\left\|\Phi_{11}^{k+1} e_{1}[0]\right\|+\lim _{k \rightarrow \infty} \sum_{i=0}^{i=k}\left\|\Phi_{11}^{i} \zeta[k-i]\right\| \\
& \leq \lim _{k \rightarrow \infty} \frac{1-\alpha^{k+1}}{1-\alpha} \bar{\zeta} \\
& \leq \frac{1}{1-\alpha} \bar{\zeta}
\end{aligned}
$$

where $\bar{\zeta}=\max \zeta[k]$. Assume $\mathcal{A}_{11}=O(1)$ and therefore $1-\alpha=O\left(T_{s}\right)$.

From (39), if $\beta_{1}[k]=O\left(T_{s}^{2}\right), \zeta[k]=O\left(T_{s}^{2}\right)$, which implies that

$$
\lim _{k \rightarrow \infty} e_{1}[k]=O\left(T_{s}\right) .
$$

It is easy to verify the dynamics of $e_{2}[k]$ can be rewritten as

$$
e_{2}[k+1]=w[k]+\theta[k]
$$

where

$$
\theta[k]=\Phi_{21} e_{1}[k]-T_{s} \overline{\mathcal{F}} f[k+0.5]-T_{s}^{3} \delta d_{2}[k]-\beta_{2}[k] .
$$

Note in (42) $\theta[k]$ involves unknown variables.

Remark 3. Note that in the continuous case, $e_{2}[k]$ would be forced to converge to 0 in finite time using a nonlinear switching injection parameter and, therefore, a sliding motion takes place. However, this is not realisable for sampled-data systems due to the sampling/hold process. However, $e_{2}[k]$ can be only driven as close to 0 as possible in 'a quasi-sliding mode'.

Let the injection signal in (31) be defined as

$$
w[k]=-\theta[k-1]
$$

and note that the expression in (43) cannot be used explicitly because $\theta[k-1]$ is unknown. However from (41) and (43), it is easy to verify

$$
w[k]=w[k-1]-e_{2}[k] .
$$

The dynamics in the system above is realisable since $e_{2}[k]$ is known and represents the 
output estimation error. From (42), it follows that

$$
\begin{aligned}
e_{2}[k+1]= & \theta[k]-\theta[k-1] \\
= & \Phi_{21}\left(e_{1}[k]-e_{1}[k-1]\right)-T_{s} \overline{\mathcal{F}}(f[k+0.5]-f[k-0.5]) \\
& -T_{s}^{3}\left(\delta d_{2}[k]-\delta d_{2}[k-1]\right)-\left(\beta_{2}[k]-\beta_{2}[k-1]\right) .
\end{aligned}
$$

Because by definition in (24)

$$
f[k+0.5]=f[k-0.5]+\frac{1}{2} \int_{(k-1) T_{s}}^{(k+1) T_{s}} v(\sigma) d \sigma
$$

and $v(t)=\dot{f}(t)$ is bounded,

$$
f[k+0.5]-f[k-0.5]=O\left(T_{s}\right) .
$$

The error dynamics in (33) therefore becomes

$$
e_{1}[k+1]-e_{1}[k]=T_{s}\left(\mathcal{A}_{11}+T_{s} \overline{\overline{\mathcal{A}}}_{11}\right) e_{1}[k]+\zeta[k] .
$$

It was shown using (40), if $\beta_{1}[k]=O\left(T_{s}^{2}\right), e_{1}[k]=O\left(T_{s}\right)$, and hence, at steady state, (47) implies that $e_{1}[k+1]-e_{1}[k]=O\left(T_{s}^{2}\right)$.

Consequently, from (45), (46), (47), if $\beta_{2}[k]=O\left(T_{s}^{2}\right)$,

$$
e_{2}[k+1]=O\left(T_{s}^{2}\right) .
$$

This expression reveals that the output estimation error is kept in a boundary layer of the output sliding plane within an order of $O\left(T_{s}^{2}\right)$. Note that since $w[k]$ evolves from the dynamics in (44), to investigate the dynamics of the estimation error overall, we essentially add the dynamics of $w[k]$ to that of $e_{1}[k]$ and $e_{2}[k]$. From (33), (34), (35), and (42), the estimation error dynamics overall are described as

$$
\begin{aligned}
e_{1}[k+1] & =\Phi_{11} e_{1}[k]-T_{s}^{2} \overline{\overline{\mathcal{F}}}_{1} f[k+0.5]-T_{s}^{3} \delta d_{1}[k]-\beta_{1}[k] \\
e_{2}[k+1] & =\Phi_{21} e_{1}[k]+w[k]-T_{s} \overline{\mathcal{F}}_{2} f[k+0.5]-T_{s}^{3} \delta d_{2}[k]-\beta_{2}[k] \\
w[k+1] & =-\Phi_{21} e_{1}[k]+T_{s} \overline{\mathcal{F}}_{2} f[k+0.5]+T_{s}^{3} \delta d_{2}[k]+\beta_{2}[k] .
\end{aligned}
$$

\subsection{Convergence analysis}

In this subsection, the convergence of the estimator (31) is first analysed when the unknown input signal and the uncertainty are not present in (1). In this particular case, the estimation error dynamics in (49) reduce to the discrete linear system

$$
\left[\begin{array}{c}
e_{1}[k+1] \\
e_{2}[k+1] \\
w[k+1]
\end{array}\right]=\left[\begin{array}{ccc}
\Phi_{11} & 0 & 0 \\
\Phi_{21} & 0 & I_{m} \\
-\Phi_{21} & 0 & 0
\end{array}\right]\left[\begin{array}{c}
e_{1}[k] \\
e_{2}[k] \\
w[k]
\end{array}\right]
$$

As seen from (50), the spectrum of the system matrix in (50) includes the eigenvalues of $\Phi_{11}$ and $\{0\}$. Because the spectrum of $\Phi_{11}$ is in the unit circle (as explained in 
the previous subsection), the spectrum of the system matrix of the estimation error dynamics in (50) is also in the unit circle. Consequently the error vectors $e_{1}[k]$ and $e_{2}[k]$ converge to 0 exponentially in the absence of disturbances and uncertainty. The results in this subsection can be encapsulated by the theorem below:

Theorem 3.1. Under Assumption 1, the estimator in (31) guarantees that the state estimates exponentially converge to their true values in the absence of the disturbance and uncertainty.

Proof: The proof is obtained from the above analysis.

\subsection{Reconstruction of the external disturbance}

In this subsection, the case when the external unknown input and uncertainty affects the system dynamics is studied, i.e. in the general error system in (49). Equation (18) implies that

$$
\Phi_{21}=O\left(T_{s}\right) .
$$

As shown in (40), $e_{1}[k]=O\left(T_{s}\right)$ if $\beta_{1}[k]=O\left(T_{s}^{2}\right)$. Thus, if $\beta_{2}[k]=O\left(T_{s}^{2}\right)$, equation (49) implies that

$$
w[k]=T_{s} \overline{\mathcal{F}}_{2} f[k-0.5]+O\left(T_{s}^{2}\right),
$$

and therefore, $f[k-0.5]$ can be inferred from $w[k]$. Because

$$
f[k]-f[k-0.5]=\frac{1}{2} \int_{(k-1) T_{s}}^{k T_{s}} v(\sigma) d \sigma=O\left(T_{s}\right),
$$

$f[k]$ can be approximated by $f[k-0.5]$ if the sampling time period $T_{s}$ is sufficiently small. Equation (51) means that

$$
w[k] \approx T_{s} \overline{\mathcal{F}}_{2} f[k-0.5]
$$

and therefore,

$$
f[k] \approx f[k-0.5] \approx \frac{\overline{\mathcal{F}}_{2}^{\dagger}}{T_{s}} w[k]
$$

where $\overline{\mathcal{F}}_{2}^{\dagger}$ is the left pseudo-inverse of $\overline{\mathcal{F}}_{2}$. The results just described can be summarized by the theorem below:

Theorem 3.2. In the presence of an unknown input signal $f[k]$ and uncertainty, if Assumptions 1 and 2 are satisfied and $\beta[k]=O\left(T_{s}^{2}\right)$, the state estimation errors are guaranteed to be $O\left(T_{s}\right)$, and the external disturbance signal is reconstructed as $f[k] \approx \frac{\overline{\mathcal{F}}_{2}^{\dagger}}{T_{s}} w[k]$ where $w[k]$ is determined in (44).

Proof: The theorem is directly proved from the above analysis. 


\section{Numerical example}

In this section, we illustrate our proposed method by using the VTOL aircraft model model in (Tan \& Edwards, 2003). For this system, the state vector contains horizontal and vertical velocities, pitch rate and angle. The output vector of the model represents horizontal and vertical velocities, and pitch angle. The control input vector includes collective and longitudinal cyclic pitch control inputs. From (Tan \& Edwards, 2003), the system matrices in (1) are:

$$
\begin{gathered}
A=\left[\begin{array}{cccc}
-9.9477 & -0.7476 & 0.2632 & 5.0337 \\
52.1659 & 2.7452 & 5.5532 & -24.4221 \\
26.0922 & 2.6361 & -4.1975 & -19.2774 \\
0 & 0 & 1.0000 & 0
\end{array}\right], \\
B=F=\left[\begin{array}{cc}
0.4422 & 0.1761 \\
3.5446 & -7.5922 \\
-5.5200 & 4.4900 \\
0 & 0
\end{array}\right], \quad C=\left[\begin{array}{llll}
1 & 0 & 0 & 0 \\
0 & 1 & 0 & 0 \\
0 & 0 & 0 & 1
\end{array}\right], \\
M=\left[\begin{array}{llll}
0 & 0 & 1 & 0
\end{array}\right]^{T} .
\end{gathered}
$$

The parametric uncertainty $\xi$ was chosen as

$$
\xi=\left[\begin{array}{lll}
0 & \Delta_{32} & \Delta_{34}
\end{array}\right] y
$$

where $\Delta_{32}=0.05$ and $\Delta_{34}=0.02$. It is easy to check that $C F$ has full rank and it can be shown that the triple $(A, F, C)$ does not have any invariant zeros. Thus, our proposed scheme for the VTOL aircraft model model is applicable. The system matrices can be converted to the structure in (1) by using the transformation below:

$$
\mathcal{T}_{0}=\left[\begin{array}{cccc}
-6.5287 & -0.7428 & -1.0000 & 0 \\
0 & 0 & 0 & 1.0000 \\
1.0000 & 0 & 0 & 0 \\
0 & 1.0000 & 0 & 0
\end{array}\right]
$$

Using the computation approach described in (Tan \& Edwards, 2003), a candidate for matrix $L$ is as follows:

$$
L=\left[\begin{array}{lll}
3.1251 & 0 & 0
\end{array}\right] .
$$

The observer (31) and the unknown input estimator (52) for the VTOL aircraft model is studied in two scenarios. First, when there is no uncertainty, and in the second case, when the uncertainty is introduced.

Because the eigenvalues of the state transition matrix $A$ lie in the left half of the complex plane, it is not necessary to employ a controller for stabilising the system. In this paper, we choose the sampling time period as $T_{s}=0.05 \mathrm{~s}$. The control signals are 
given as

$$
\begin{aligned}
& u_{1}[k]=0.1+0.1 \sin \left(0.1 T_{s} k\right) \\
& u_{2}[k]=0.1 \sin \left(0.5 T_{s} k+\pi / 2\right) .
\end{aligned}
$$

The external disturbance functions are chosen as

$$
\begin{aligned}
f_{1}(t) & =0.1+2 \sin (t) \\
f_{2}(k) & =-0.5+\sin (t-\pi / 2) .
\end{aligned}
$$

Fig. 1 shows the evolution of the system states and their estimates when there is no uncertainty. It is observed that the estimates converge to their true values quickly. The estimate of $x_{3}$ is the slowest to converge to its true value at around $0.8 \mathrm{~s}$. Fig. 3 shows the quasi-sliding motion of the error dynamics in which the evolution of the output error lies in a $O\left(T_{s}^{2}\right)$ boundary layer around the origin. Fig. 5 reveals the closeness of the estimate of the external signals to their true values.

In the presence of uncertainty, Fig. 2 shows the evolution of the system states and their estimates. Here, the convergence speed of the estimate of $x_{3}$ is slower than the others and the performance (as predicted) deteriorates because of the influence of the uncertainty. Fig. 4 shows a quasi-sliding motion which occurs in an $O\left(T_{s}^{2}\right)$ boundary layer around the origin. In Fig. 5, the estimates of the unknown input signals are close to their true values.
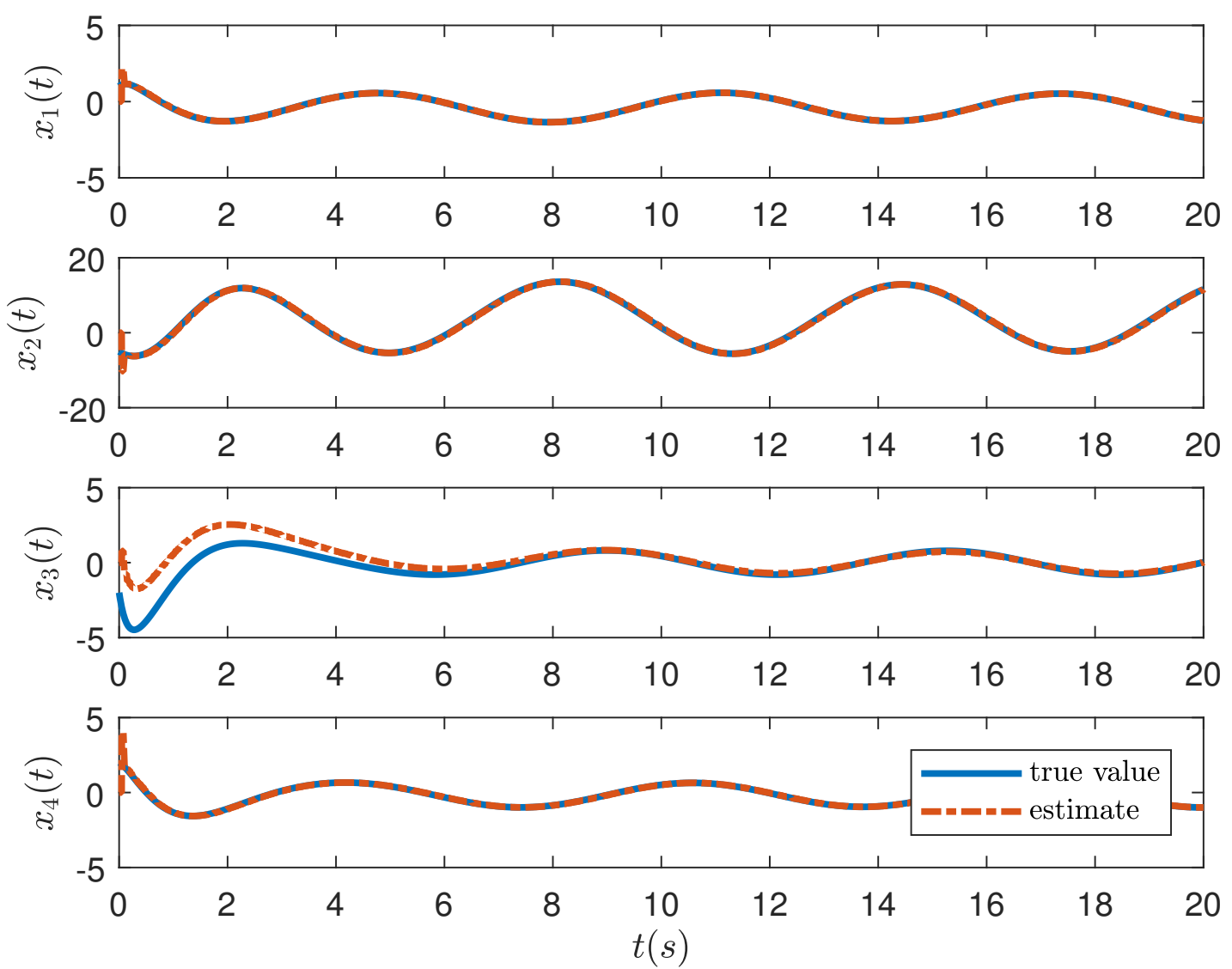

Figure 1. Evolution of the state variables and their estimates for the case without the uncertainty 

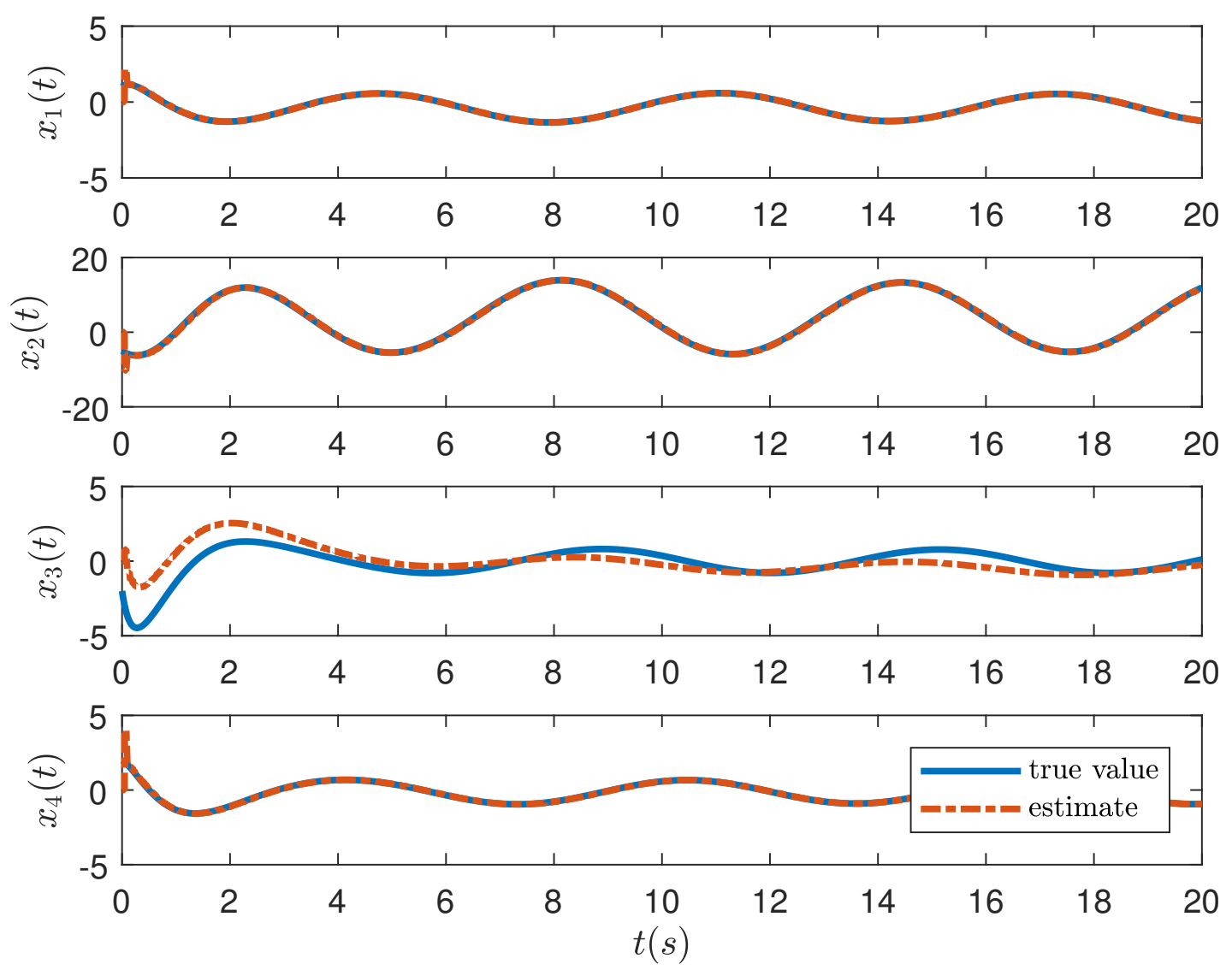

Figure 2. Evolution of the state variables and their estimates for the case with the uncertainty

To illustrate the effectiveness of our proposed scheme, we simulated the same scenarios using the continuous time sliding mode observer presented in (Tan \& Edwards, 2003), implemented with a sample time of 0.05 s. Figs. 6 and 7 show that the output errors are worse than those using the proposed observer. In addition, Fig. 8 reveals that the observer in (Tan \& Edwards, 2003) gives poor results in terms of estimating the unknown external signals.

In this section, the numerical simulations have elucidated our theoretical analysis and shown that our method is able to reliably recover the system states and unknown inputs for sampled-data systems in the presence of uncertainty.

\section{CONCLUSION}

In this paper, a new observer has been proposed to simultaneously estimate the system states and unknown inputs in a class of sampled-data systems subject to external signals and uncertainties. Our proposed scheme is constructed based on quasi-sliding motion ideas, although its derivation is taken from the design for the continuous time version. A formal analysis of stability and robustness has been provided and simulation results illustrated the effectiveness of the proposed method. The observer in this paper is applicable for systems which satisfy the rank condition in Assumption 1. A future topic of research will seek to address the observer design for a more general class of systems. 

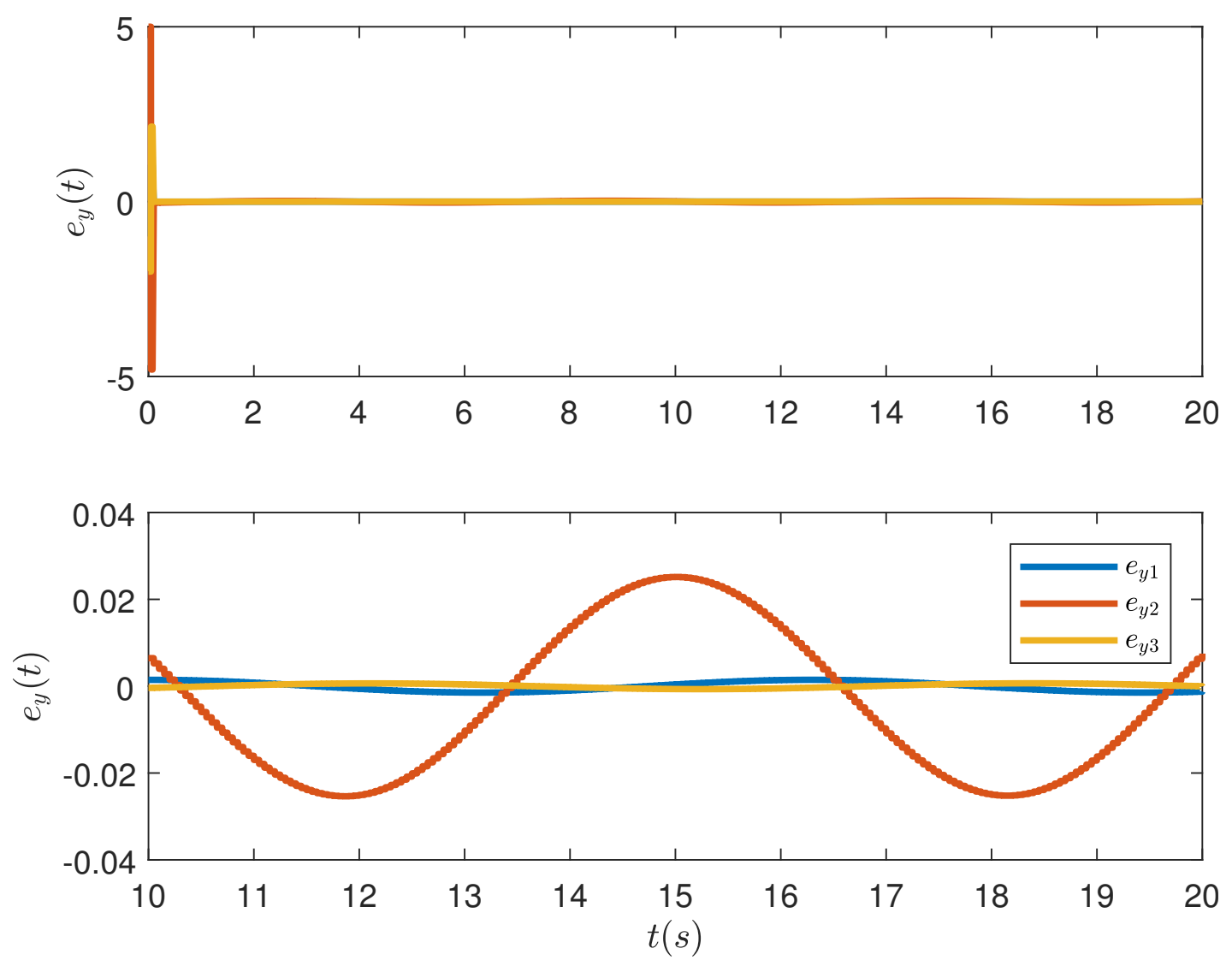

Figure 3. Evolution of the output errors for the case without the uncertainty

\section{References}

Alwi, H., \& Edwards, C. (2008). Fault tolerant control using sliding modes with on-line control allocation. Automatica, 44 (7), 1859 - 1866.

Bejarano, F. J., \& Fridman, L. (2010). High order sliding mode observer for linear systems with unbounded unknown inputs. International Journal of Control, 83(9), 1920-1929.

Chakrabarty, A., Ayoub, R., Żak, S. H., \& Sundaram, S. (2017). Delayed unknown input observers for discrete-time linear systems with guaranteed performance. Systems $\mathcal{E}$ Control Letters, 103, 9 - 15.

Chandra, K. P. B., Alwi, H., \& Edwards, C. (2017). Fault detection in uncertain lpv systems with imperfect scheduling parameter using sliding mode observers. European Journal of Control, 34, 1 - 15 .

Charandabi, B. A., \& Marquez, H. J. (2014). A novel approach to unknown input filter design for discrete-time linear systems. Automatica, 50(11), 2835 - 2839.

Darouach, M., Zasadzinski, M., \& Xu, S. J. (1994, March). Full-order observers for linear systems with unknown inputs. IEEE Transactions on Automatic Control, 39(3), 606-609.

Edwards, C., Spurgeon, S. K., \& Patton, R. J. (2000). Sliding mode observers for fault detection and isolation. Automatica, 36(4), 541 - 553.

Floquet, T., \& Barbot, J.-P. (2006). State and unknown input estimation for linear discretetime systems. Automatica, 42(11), 1883 - 1889.

Floquet, T., Edwards, C., \& Spurgeon, S. K. (2007). On sliding mode observers for systems with unknown inputs. International Journal of Adaptive Control and Signal Processing, $21(8-9), 638-656$. 

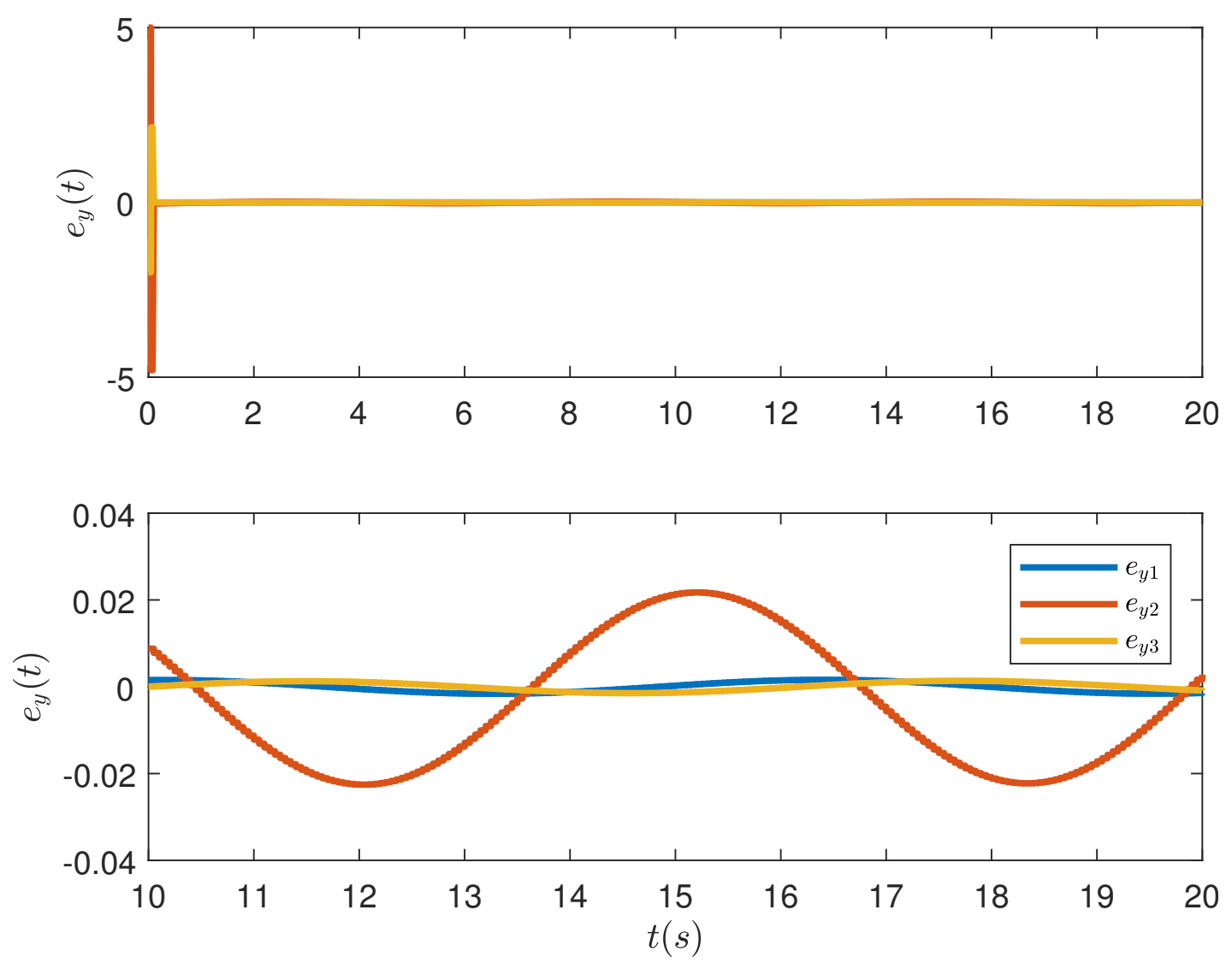

Figure 4. Evolution of the output errors for the case with the uncertainty

Fridman, L., Levant, A., \& Davila, J. (2007). Observation of linear systems with unknown inputs via high-order sliding-modes. International Journal of Systems Science, 38(10), 773-791. Retrieved from https://doi.org/10.1080/00207720701409538

Gucik-Derigny, D., Raïssi, T., \& Zolghadri, A. (2016). A note on interval observer design for unknown input estimation. International Journal of Control, 89(1), 25-37.

Han, X., Fridman, E., \& Spurgeon, S. (2014). Sampled-data sliding mode observer for robust fault reconstruction: A time-delay approach. Journal of the Franklin Institute, 351 (4), 2125 - 2142. (Special Issue on 2010-2012 Advances in Variable Structure Systems and Sliding Mode Algorithms)

Kokotovic, P., Khalil, H., \& O'Reilly, J. (1986). Singular perturbation methods in control: Analysis and design. Orlando, FL: Academic Press.

Levant, A. (2011). Discretization issues of high-order sliding modes. IFAC Proceedings Volumes, 44(1), 1904 - 1909. Retrieved from http://www.sciencedirect.com/science/article/pii/S1474667016438899 (18th IFAC World Congress)

Livne, M., \& Levant, A. (2014). Proper discretization of homogeneous differentiators. Automatica, 50(8), 2007 - 2014. Retrieved from http://www.sciencedirect.com/science/article/pii/S0005109814002180

Marro, G., Bernstein, D. S., \& Zattoni, E. (2010, Dec). Geometric methods for unknownstate, unknown-input reconstruction in discrete-time nonminimum-phase systems with feedthrough terms. In 49th IEEE Conference on Decision and Control (CDC) (p. 6022$6027)$.

Marro, G., Zattoni, E., \& Bernstein, D. S. (2011). Geometric insight and structure algorithms 

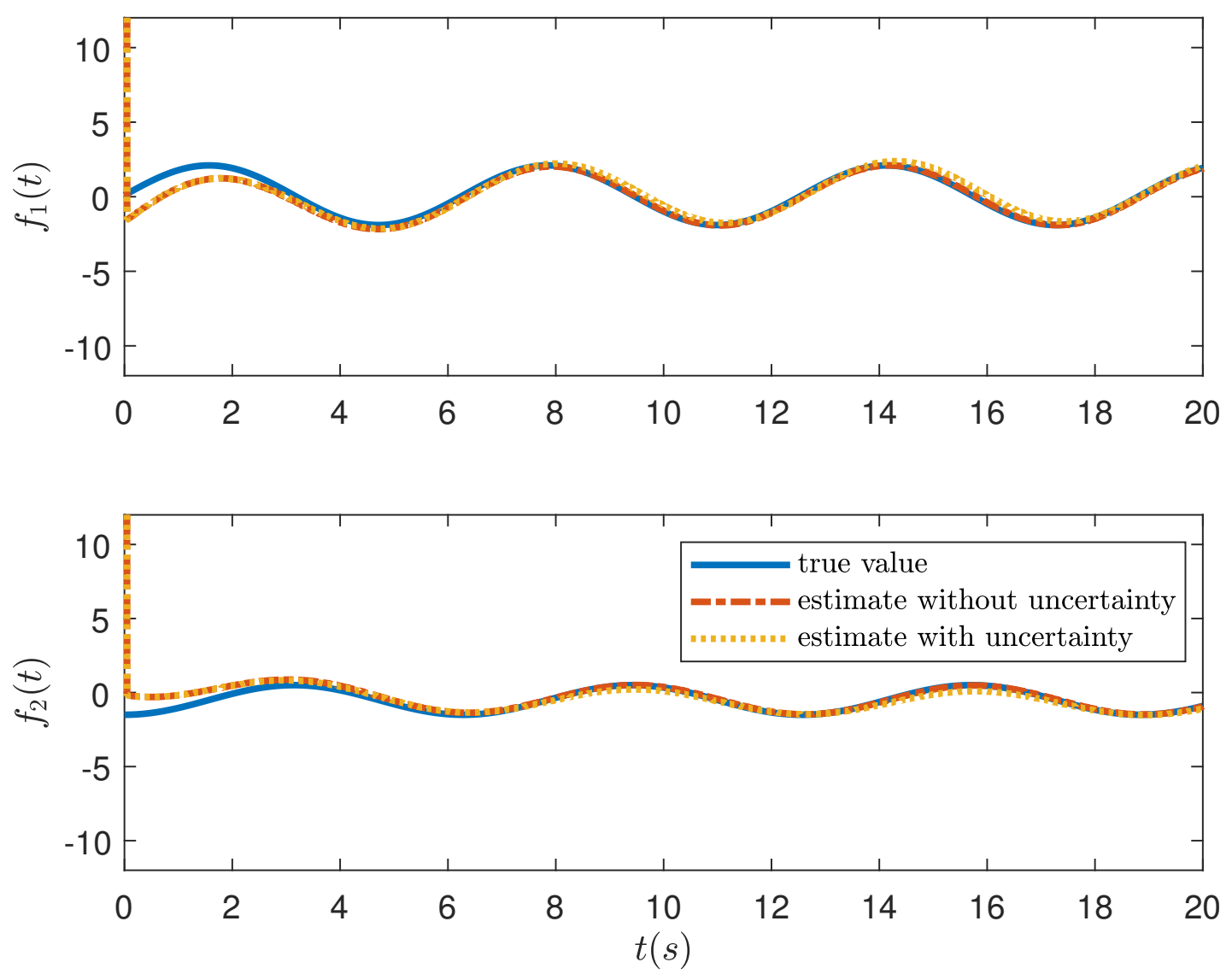

Figure 5. Evolution of the external signals and their estimates

for unknown-state, unknown-input reconstruction in linear multivariable systems. IFAC Proceedings Volumes, 44(1), 11320 - 11325. (18th IFAC World Congress)

Milosavljevic, C. (1985). General conditions for the existence of quasi-sliding mode on the switching hyper-plane in discrete variable structure systems. Automat. Remote Contr., 46(3), 307-314.

Nguyen, T., Edwards, C., Azimi, V., \& Su, W.-C. (2019). Improving control effort in output feedback sliding mode control of sampled-data systems. IET Control Theory \& Applications, doi 10.1049/iet-cta.2018.5080.

Nguyen, T., Su, W.-C., \& Gajic, Z. (2010, June 30-July 02). On $O\left(T^{2}\right)$ state regulation with output feedback sliding mode control for sampled-data systems. In Proc. of the american control conference (p. 1823-1828). Baltimore, USA.

Nguyen, T., Su, W. C., Gajic, Z., \& Edwards, C. (2016, Oct). Higher accuracy output feedback sliding mode control of sampled-data systems. IEEE Transactions on Automatic Control, $61(10), 3177-3182$.

Sundaram, S., \& Hadjicostis, C. N. (2007, Feb). Delayed observers for linear systems with unknown inputs. IEEE Transactions on Automatic Control, 52(2), 334-339.

Tan, C. P., \& Edwards, C. (2003). Sliding mode observers for robust detection and reconstruction of actuator and sensor faults. International Journal of Robust and Nonlinear Control, $13(5), 443-463$.

Tan, C. P., \& Edwards, C. (2010, April). Robust fault reconstruction in uncertain linear systems using multiple sliding mode observers in cascade. IEEE Transactions on Automatic Control, 55(4), 855-867.

Yan, X.-G., \& Edwards, C. (2007). Nonlinear robust fault reconstruction and estimation using 

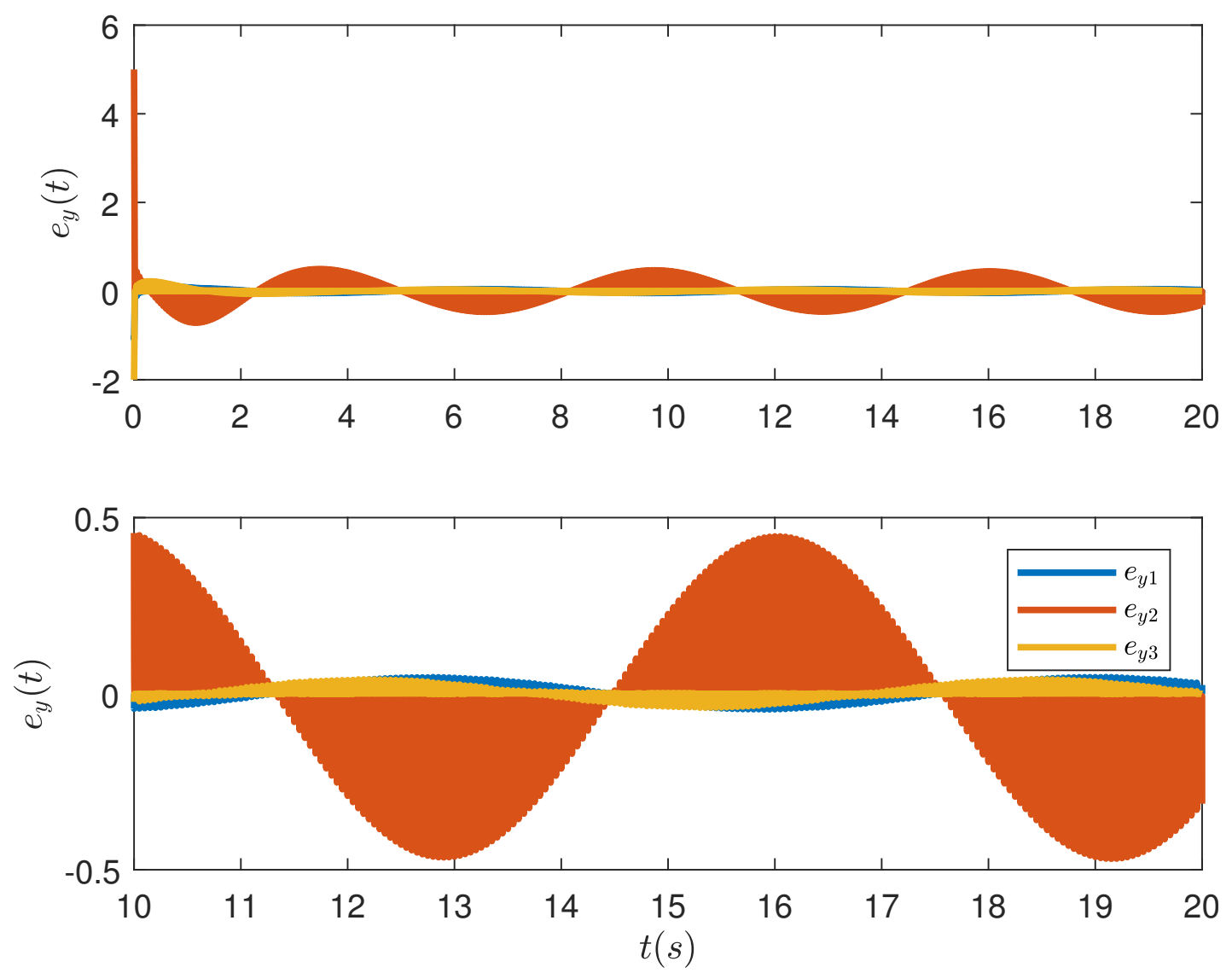

Figure 6. Evolution of the output errors for the case without the uncertainty using the sliding mode observer in (Tan \& Edwards, 2003).

a sliding mode observer. Automatica, 43(9), 1605 - 1614.

\section{Appendix A. Proof of Lemma 2.1}

The proof follows the arguments in (Nguyen et al., 2016). The expression in (25a) is a modified version of that in (Nguyen et al., 2016) and the remainder of the lemma follows the proof in (Nguyen et al., 2016). It is easy to verify that

$$
\begin{aligned}
f[k+1] & =f[k]+\int_{k T_{s}}^{(k+1) T_{s}} v(\sigma) d \sigma \\
& =f[k]+\int_{k T_{s}}^{(k+1) T_{s}}\left(v[k]+\int_{k T_{s}}^{\sigma} \dot{v}(\tau)\right) d \sigma d \tau .
\end{aligned}
$$

and this implies

$$
v[k]=\frac{f[k+1]-f[k]}{T_{s}}-\frac{1}{T_{s}} \int_{k T_{s}}^{(k+1) T_{s}} \int_{k T_{s}}^{\sigma} \dot{v}(\tau) d \sigma d \tau .
$$



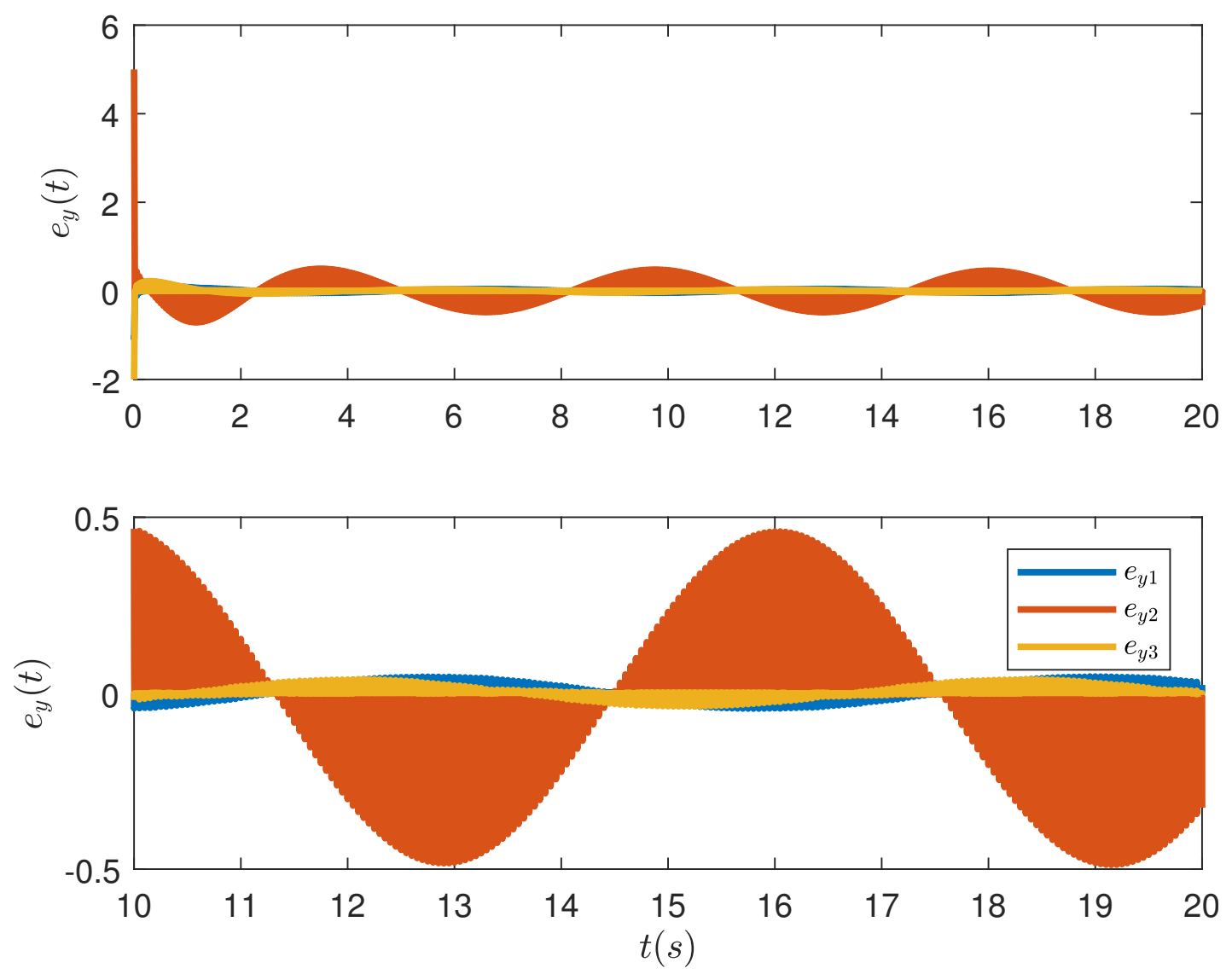

Figure 7. Evolution of the output errors for the case with the uncertainty using the sliding mode observer in (Tan \& Edwards, 2003).

Hence,

$$
\begin{aligned}
d[k] & =\Pi f[k]+\frac{T_{s}}{2} \Pi v[k]+T_{s}^{3} \Delta d_{0}[k] \\
& =\Pi f[k]+\frac{T_{s}}{2} \Pi \frac{f[k+1]-f[k]}{T_{s}}-\frac{\Pi}{2} \int_{k T_{s}}^{(k+1) T_{s}} \int_{k T_{s}}^{\sigma} \dot{v}(\tau) d \sigma d \tau+T_{s}^{3} \Delta d_{0}[k] \\
& =\Pi \frac{f[k+1]+f[k]}{2}-\frac{\Pi}{2} \int_{k T_{s}}^{(k+1) T_{s}} \int_{k T_{s}}^{\sigma} \dot{v}(\tau) d \sigma d \tau+T_{s}^{3} \Delta d_{0}[k] \\
& =\Pi f\left[k+\frac{1}{2}\right]+T_{s}^{3} \Delta d[k]
\end{aligned}
$$

as claimed. 

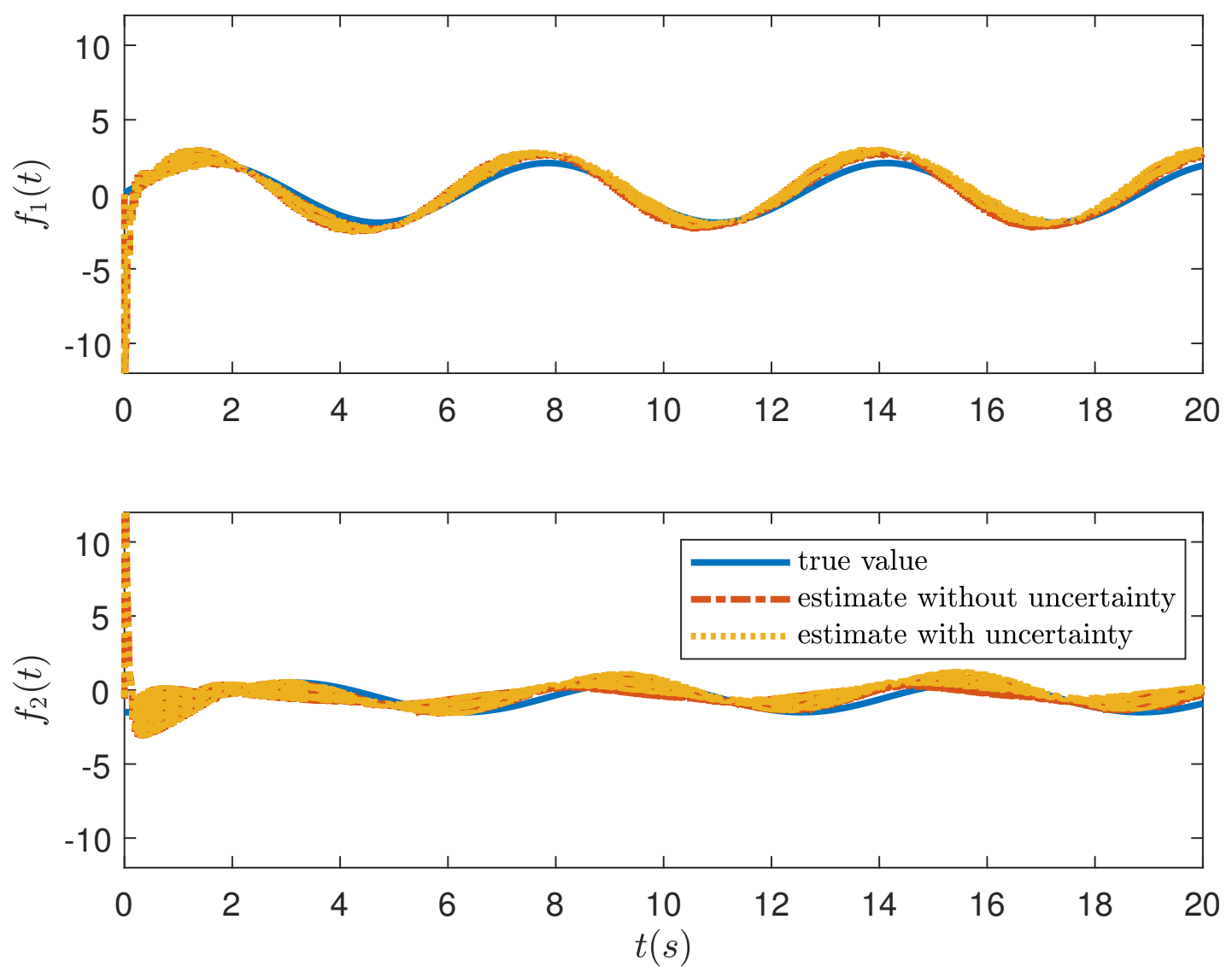

Figure 8. Evolution of the external signals and their estimates using the sliding mode observer in (Tan \& Edwards, 2003). 